\section{Autopercepção da mastigação e fatores associados em adultos brasileiros}

\author{
Poor self-rated mastication and associated \\ factors in Brazilian adults
}

\author{
${ }^{1}$ Faculdade de Medicina, \\ Universidade Federal de \\ Minas Gerais, Belo Horizonte, \\ Brasil. \\ 2 Faculdade de Odontologia, \\ Universidade Estadual \\ de Montes Claros, Montes \\ Claros, Brasil. \\ Correspondência \\ A. P. G. Braga \\ Departamento de Clínica \\ Médica, Faculdade de \\ Medicina, Universidade \\ Federal de Minas Gerais. \\ Rua Pintor Vicente de Abreu \\ 100, apto. 301, Belo Horizonte, \\ MG 30450-610, Brasil. \\ anapaulagasparini@yahoo. \\ com.br
}

\section{Abstract}

This study estimated the prevalence of bad/very bad self-rated mastication and investigated associated factors among Brazilian adults. The sample included 13,431 adults examined and interviewed in the SBBrazil project. Self-rated mastication was combined in three categories: good/ very good (the reference), fair, and bad/very bad. Multinomial logistic regression was performed to measure the association between fair and bad/ very bad self-rated mastication and all independent variables included in the analysis, with significance set at $p<0.05 .55 .6 \%$ of participants rated their mastication as good/very good, $24.9 \%$ as fair, and $19.5 \%$ as bad/very bad. In the final multivariate analysis, factors associated with bad or fair self-rated mastication were: receiving no information on how to avoid oral problems; presenting fewer than 23 remaining teeth; requiring total or partial prosthesis; bad/very bad self-rated oral health; gingival pain and/or toothache in the previous year; and need of dental treatment. In conclusion, various factors were associated with self-rated mastication, especially subjective conditions, thus emphasizing its importance as an oral health indicator.

Mastication; Oral Health; Adult
Ana Paula Gasparini Braga 1

Sandhi Maria Barreto 1

Andréa Maria Eleutério de Barros Lima Martins 2

\section{Introdução}

A mastigação é uma das funções mais importantes do sistema estomatognático por relacionar-se à nutrição, ao crescimento e ao desenvolvimento craniofacial, à maturação da musculatura orofacial, à estabilidade oclusal e à estabilidade da articulação temporomandibular 1,2,3,4. O padrão e a habilidade mastigatória podem ser influenciados por disfunções temporomandibulares, dor, distúrbios miofuncionais orofaciais, má oclusão, perdas dentárias, uso de próteses mal adaptadas, presença de cárie e/ou doença periodontal 1,2,3,4,5,6. As dificuldades de mastigação, deglutição e/ou fala podem acarretar um quadro de incapacidade bucal transitória ou permanente com grau, abrangência e evolução variando de indivíduo para indivíduo, segundo o tempo, as características clínicas, as possibilidades terapêuticas e a inserção social 6,7,8,9,10. O termo "incapacidade bucal" refere-se à presença de limitações em certas atividades diárias, como dificuldade de consumir alimentos muito fibrosos ou duros e incômodo ao alimentar-se em frente a outras pessoas 7,8 .

A insatisfação mastigatória é um importante indicador de incapacidade bucal ${ }^{8}$. Gilbert et al. 7 realizaram um estudo na Flórida, Estados Unidos, e verificaram que, aproximadamente, $16 \%$ dos adultos relataram tal insatisfação, sendo essa associada a fatores clínicos diagnosticados ou referidos como doença bucal (cáries 
dentárias, gengivite, doença periodontal, ausência de dentes, mobilidade dental, abscesso, fraturas dentárias, lesões de boca e xerostomia), dor, limitação funcional (dificuldades na fala e mastigação) e incapacidade bucal. No Brasil, alguns estudos populacionais abordaram a mastigação como item da saúde bucal 11,12, tendo sido verificada a associação entre a insatisfação com a mastigação e a piora da saúde bucal e da qualidade de vida 11. Foi identificado apenas um estudo, entre idosos, sobre os fatores associados à autopercepção da mastigação no país 6 . Nesse estudo, utilizando dados do inquérito SBBrasil: Condições de Saúde Bucal na População Brasileira, a prevalência de mastigação insatisfatória em idosos foi de $49,7 \%$, sendo maior entre os que apresentaram necessidade de prótese dentária, cárie dentária, doença periodontal e relato de dor em dentes ou gengivas 6 .

Ainabilidademastigatória também pode estar associada à mortalidade e a doenças cardiovasculares 13,14. Um estudo de coorte verificou que a redução na habilidade mastigatória aumentou o risco de mortalidade em idosos e concluiu que a habilidade mastigatória pode predizer, de forma independente, uma maior sobrevida 13.

A mastigação pode ser avaliada tanto normativamente, pelo profissional de saúde, como subjetivamente, pelo próprio indivíduo. Entretanto, a literatura aponta haver divergências entre esses dois métodos de avaliação sobre tais condições 6,12. Não foram identificados estudos de base populacional sobre a autopercepção da mastigação da população adulta brasileira. Assim, o presente artigo tem por objetivo conhecer a prevalência da autopercepção da mastigação como boa, regular e ruim e identificar os fatores associados às classificações da autopercepção da mastigação como regular e ruim, utilizando um modelo multidimensional que considera o ambiente externo, as características individuais, os comportamentos relacionados à saúde, as condições normativas e subjetivas de saúde bucal 12 .

\section{Métodos}

\section{Delineamento do estudo}

Estudo transversal que utilizou a base de dados do inquérito SBBrasil: Condições de Saúde Bucal na População Brasileira, realizado pelo Ministério da Saúde, nos anos 2002-2003 15.

\section{População do estudo}

O SBBrasil incluiu indivíduos residentes em zonas urbanas e rurais de 250 municípios, em todos os estados brasileiros, de seis estratos etários populacionais (18-36 meses, 5 anos, 12 anos, 15-19 anos, 35-44 anos e 65-74 anos). O tamanho da amostra foi calculado para cada macrorregião a partir das estimativas de ataque de cárie produzidas em 1986. A técnica de amostragem foi probabilística por conglomerados, sendo os indivíduos selecionados por meio de sorteio, considerando o porte populacional dos municípios. Foram sorteados dez municípios em cada estrato de porte populacional, perfazendo um total de 50 municípios por região 15 . No presente estudo, foram utilizados os dados referentes aos 13.431 adultos (35-44 anos) participantes do SBBrasil.

\section{Procedimentos}

Os dados do inquérito foram coletados por cirurgiões-dentistas treinados e calibrados (concordância kappa ou percentual), nos domicílios dos participantes, por meio de entrevista estruturada e exame da cavidade bucal. Os cirurgiões-dentistas foram orientados a repetir o treinamento quando os resultados do teste kappa ou da concordância percentual não fossem satisfatórios. Cerca de 5\% dos exames foram executados em duplicata com o objetivo de mensurar a concordância intraexaminador. Os exames foram realizados com a utilização de instrumentos preconizados pela Organização Mundial da Saúde (sonda periodontal, espelho bucal plano e espátulas de madeira), sob iluminação natural. A coleta de dados foi conduzida conforme os princípios éticos da Declaração de Helsinki e da Resolução $n^{\circ}$. 196/96 do Conselho Nacional de Saúde e aprovada pela Comissão Nacional de Ética em Pesquisa (parecer $n^{\circ} .581 / 2000$ ). Maiores detalhes metodológicos podem ser encontrados em outra publicação 15 .

\section{Variáveis do estudo}

A variável dependente foi a autopercepção da mastigação que foi obtida por meio das respostas à seguinte pergunta: "Como classificaria sua mastigação?” (ótima, boa, regular, ruim, péssima). As respostas foram agregadas em três categorias: ótima/boa, regular e ruim/péssima, denominadas como "boa", "regular" e "ruim".

As variáveis independentes foram agrupadas seguindo orientação proposta pelo modelo de Gift et al. 16, adaptado por Martins et al. 12. O modelo é apresentado na Figura 1 e contempla a interação entre fatores do ambiente externo, características individuais, comportamentos relacionado à saúde, condições normativas e subjetivas de saúde bucal. 


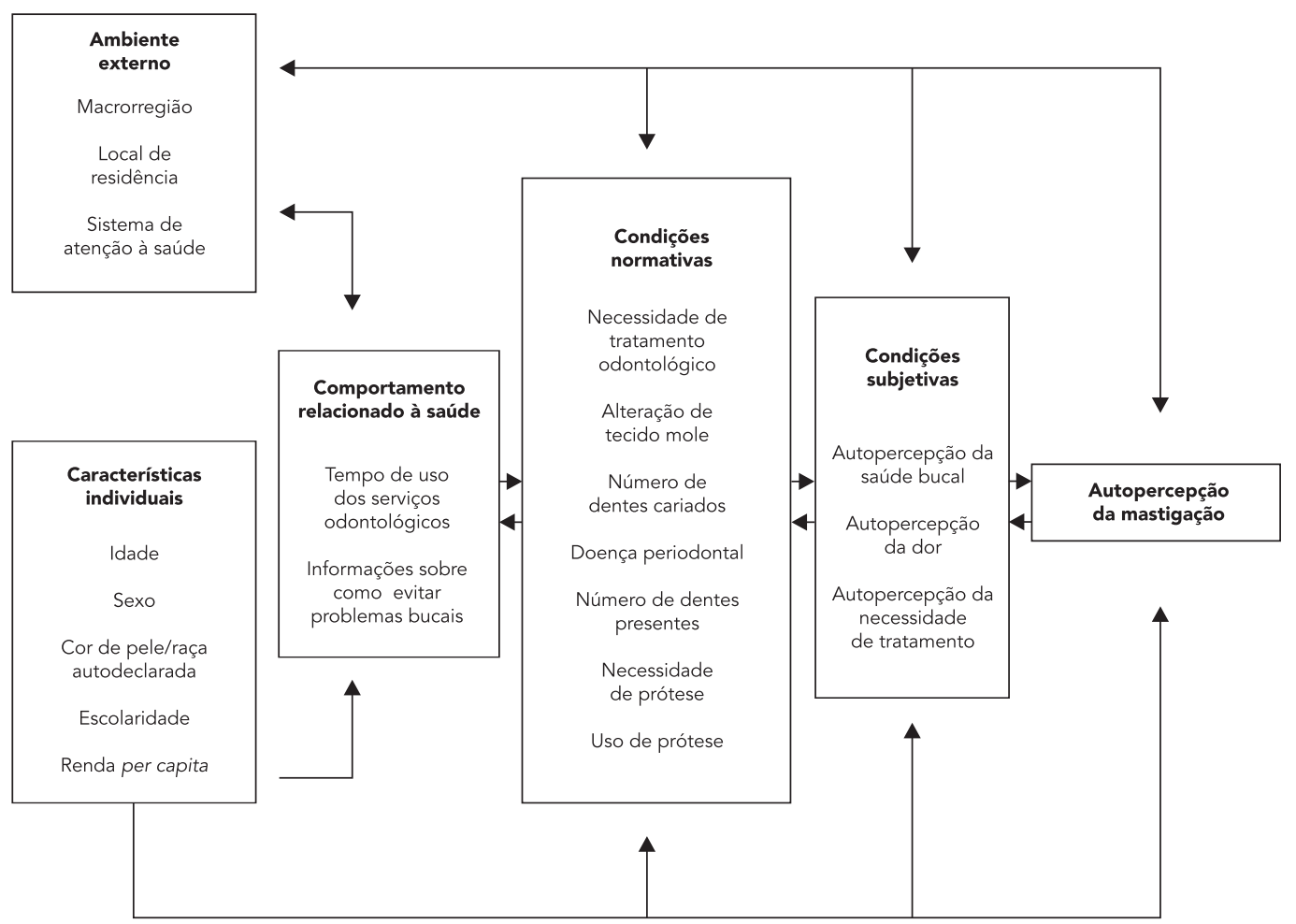

As variáveis referentes ao ambiente externo foram: macrorregião do Brasil (Sul, Sudeste, Centro-oeste, Nordeste, Norte), local de residência (zona urbana, zona rural) e sistema de atenção à saúde utilizado (plano de saúde/particular, público/filantrópico). As variáveis que compõem o bloco de características individuais foram as sociodemográficas: sexo (feminino ou masculino), idade em anos (35-39 e 40-44), cor da pele/raça autodeclarada (branco, pardo, negro, amarelo/ indígena), escolaridade - em anos de estudo (8 anos ou mais, de 4-7 anos e de 0-3 anos) e renda per capita - em percentual do salário mínimo $(\geq 68 \%, \geq 31 \%$ e $<68 \%$ e $<31 \%$ ) e os valores equivalentes em dólar ( $\geq$ US\$50,00, $\geq$ US $\$ 22,33$ e $<$ US\$50,00 e < US\$22,33). Os critérios de autoclassificação de cor/raça usados no SB Brasil são os mesmos do censo demográfico do Instituto Brasileiro de Geografia e Estatística (IBGE), exceto a classificação "preto" que foi substituída por "negro", por essa última ter sido a utilizada no instrumento de coleta de dados do SB Brasil. Os comportamentos relacionados à saúde bucal foram o tempo de uso de serviços odontológicos (há 3 anos ou menos, há mais de 3 anos) e o acesso a informações sobre como evitar problemas bucais (sim ou não).

Uma das condições normativas de saúde bucal foi a necessidade de tratamento odontológico (não ou sim) construída a partir das variáveis: necessidade de tratamento dentário, necessidade de tratamento periodontal e necessidade de próteses, concomitantemente. No exame, foram avaliadas as seguintes necessidades: restauração de uma superfície dentária, restauração de duas ou mais superfícies dentárias, coroa por qualquer razão, faceta estética, tratamento pulpar e restauração, extração, remineralização de mancha branca e selante. A necessidade de tratamento periodontal foi definida com base no índice periodontal comunitário (CPI): saudável, sangramento gengival, cálculo, bolsa de 4 a $5 \mathrm{~mm}$, bolsa de mais de $6 \mathrm{~mm}$. Todos os indivíduos com escore do CPI $\geq 1$ (sangramento gengival) foram considerados como necessidade de tratamento periodontal. Quanto à necessidade de prótese, 
considerou-se a arcada superior e inferior e foi categorizada em: não necessita, necessita substituir um elemento, necessita substituir mais de um elemento ou necessita de prótese dentária total. A variável doença periodontal (não ou sim) também foi analisada isoladamente, sendo considerado "doente periodontal" o indivíduo que apresentava, num mesmo sextante, bolsa periodontal $\geq 4$ e perda de inserção $\geq 4$ (PIP $\geq 2$ ). As demais condições normativas de saúde bucal avaliadas foram: presença de alterações de tecidos moles (não ou sim); número de dentes permanentes presentes (23-32 dentes, 13-22, 3-12 e $0-2)$; número de dentes permanentes cariados (0, 1-3, 4 ou mais); necessidade de prótese removível (não necessita, necessita parcial, necessita total) e uso de prótese removível (não usa, usa parcial, usa total).

As condições subjetivas relacionadas à saúde bucal incluíram: autopercepção da saúde bucal (ótima/boa, regular, ruim/péssima), dor nos dentes e gengivas nos últimos seis meses (não ou sim) e autopercepção da necessidade de tratamento odontológico (não ou sim).

\section{Análise estatística}

Inicialmente, foi feita a descrição das variáveis a fim de caracterizar a população do estudo. Os adultos que perceberam sua mastigação como ruim ou regular foram comparados aos que a perceberam como boa em relação a cada fator de interesse ou variável independente, agrupados segundo o modelo apresentado. A associação entre cada variável e a autopercepção da mastigação foi verificada por meio do teste de qui-quadrado de Pearson $\left(\chi^{2}\right)$, com nível de significância de 0,20 . A seguir, foi realizada a análise bivariada por meio da regressão logística multinomial para verificação das magnitudes das associações entre a variável dependente e os fatores de interesse. Elas foram estimadas pela razão de chances, com nível de significância de 0,05 e respectivos intervalos de 95\% de confiança (IC95\%). Em seguida, foi realizada a análise multivariada por bloco, incluindo todas as variáveis associadas estatisticamente à variável dependente na análise bivariada. Finalmente, foi construído um modelo único, com base nos fatores retidos nos modelos intermediários e que permaneceram associados à mastigação regular ou ruim ao nível de $\mathrm{p} \leq 0,05$. As variáveis doença periodontal e número de dentes permanentes cariados não participaram da análise multivariada por estarem presentes somente no grupo de indivíduos dentados. A análise foi feita utilizando o programa Stata 9.0 (Stata Corp., College Station, Estados Unidos).

\section{Considerações éticas e conflitos de interesse}

O presente estudo foi aprovado pelo Comitê de Ética em Pesquisa da Universidade Federal de Minas Gerais (parecer número 0306.0.203.00010). Não há conflitos de interesse.

\section{Resultados}

Dos 13.431 adultos participantes do inquérito SB Brasil, 13.182 responderam à questão sobre a autopercepção da mastigação, sendo que $55,6 \%$ deles classificaram a mastigação como boa; $24,9 \%$, como regular; e 19,5\%, como ruim. A maioria dos participantes $(88,3 \%)$ residia na zona urbana; $52,4 \%$ utilizaram o sistema de saúde público ou filantrópico; $67,6 \%$ eram mulheres; $43,9 \%$ declararam a raça/cor de pele como branca; e $42,3 \%$, como parda; além disso, $74,1 \%$ possuíam mais de 4 anos de estudo. Com relação aos comportamentos relacionados à saúde bucal, mais da metade $(62,1 \%)$ visitou o dentista há 3 anos ou menos, e 55,3\% haviam recebido informações sobre como evitar os problemas bucais.

Ao se analisarem as condições normativas de saúde bucal, observou-se que grande parte dos adultos necessitava de tratamento odontológico (88\%), sendo que praticamente um quarto dos adultos (23,5\%) necessitava de prótese parcial, e uma pequena parte, de prótese total $(4,5 \%)$. Quanto às condições subjetivas, 37\% dos indivíduos perceberam a saúde bucal como regular; e $22 \%$, como ruim ou péssima; $34,7 \%$ relataram dor nos dentes e gengivas há menos de seis meses; e $79 \%$ relataram necessitar de tratamento odontológico.

Todos os fatores independentes incluídos na análise foram associados à mastigação regular ou ruim, ao nível de $\mathrm{p}<0,20$ (Tabela 1). Na análise bivariada, considerando o nível de significância de 0,05, a percepção ruim da mastigação esteve associada positivamente a: residir nas regiões Sudeste, Centro-oeste, Nordeste e Norte; ter cobertura de serviço odontológico público ou filantrópico; idade entre 40-44 anos; cor de pele autodeclarada parda ou negra; 7 anos ou menos de escolaridade; renda per capita menor que $68 \%$ do salário mínimo (<US\$50,00); ter usado serviço odontológico há mais de 3 anos; não ter recebido informações sobre como evitar os problemas bucais; necessitar de tratamento odontológico; apresentar alteração de tecido mole; ter um ou mais dentes cariados; ser portador de doença periodontal; ter menos de vinte e três dentes permanentes; necessitar de próteses parciais ou totais; perceber a saúde bucal como regular ou ruim; relatar dor nos dentes ou 


\section{Tabela 1}

Prevalência e distribuição da autopercepção da mastigação como boa, regular e ruim segundo fatores do ambiente externo, características individuais, comportamentos em saúde bucal e condições normativas e subjetivas de saúde bucal.

Projeto SBBrasil 2002-2003.

\begin{tabular}{|c|c|c|c|c|}
\hline Variáveis independentes & $\begin{array}{c}\text { Boa } \\
(n=7.322) \\
\%\end{array}$ & $\begin{array}{c}\text { Regular } \\
(\mathrm{n}=3.284) \\
\%\end{array}$ & $\begin{array}{c}\text { Ruim } \\
(\mathrm{n}=2.576) \\
\%\end{array}$ & Valor de $p$ \\
\hline \multicolumn{5}{|l|}{ Ambiente externo } \\
\hline \multicolumn{5}{|l|}{ Macrorregião } \\
\hline Sul & 27,1 & 22,2 & 16,2 & \\
\hline Sudeste & 18,0 & 17,3 & 16,4 & \\
\hline Centro-oeste & 15,7 & 14,1 & 15,4 & \\
\hline Nordeste & 22,5 & 25,4 & 31,7 & \\
\hline Norte & 16,7 & 21,0 & 20,3 & $<0,001$ * \\
\hline \multicolumn{5}{|l|}{ Local } \\
\hline Urbana & 88,3 & 89,3 & 87,1 & \\
\hline Rural & 11,7 & 10,7 & 12,9 & 0,037 * \\
\hline \multicolumn{5}{|l|}{ Sistema de atenção à saúde } \\
\hline Plano de saúde/Particular & 51,4 & 45,9 & 40,5 & \\
\hline Público/Filantrópico & 48,6 & 54,1 & 59,5 & $<0,001$ * \\
\hline \multicolumn{5}{|l|}{ Características individuais } \\
\hline \multicolumn{5}{|l|}{ Idade (anos) } \\
\hline $35-39$ & 55,0 & 53,7 & 52,0 & \\
\hline $40-44$ & 45,0 & 46,2 & 48,0 & 0,024 * \\
\hline \multicolumn{5}{|l|}{ Sexo } \\
\hline Feminino & 65,7 & 68,1 & 72,4 & \\
\hline Masculino & 34,3 & 31,8 & 27,6 & $<0,001$ * \\
\hline \multicolumn{5}{|l|}{ Cor de pele autodeclarada } \\
\hline Branco & 48,3 & 42,2 & 34,7 & \\
\hline Pardo & 38,6 & 43,9 & 50,7 & \\
\hline Negro & 8,7 & 9,3 & 10,6 & \\
\hline Amarelo e índio & 4,4 & 4,6 & 4,0 & $<0,001$ * \\
\hline \multicolumn{5}{|l|}{ Escolaridade (anos de estudo) } \\
\hline 8 ou mais & 40,2 & 38,8 & 28,3 & \\
\hline $4-7$ & 37,4 & 36,9 & 36,8 & \\
\hline $0-3$ & 22,4 & 24,3 & 34,9 & $<0,001$ * \\
\hline \multicolumn{5}{|l|}{ Renda per capita (\% do salário mínimo/dólar) } \\
\hline$\geq 68 \%(\geq$ US $\$ 50,00)$ & 42,1 & 34,3 & 25,5 & \\
\hline$\geq 31 \%$ e $<68 \%(\geq$ US $\$ 22,33$ e $<$ US $\$ 50,00)$ & 27,8 & 30,2 & 29,7 & \\
\hline$<31 \%(<$ US $\$ 22,33)$ & 30,0 & 35,4 & 44,8 & $<0,001$ * \\
\hline \multicolumn{5}{|l|}{ Comportamento } \\
\hline \multicolumn{5}{|l|}{ Tempo de uso dos serviços odontológicos (anos) } \\
\hline 3 ou menos & 64,9 & 63,5 & 52,9 & \\
\hline Mais de 3 & 35,1 & 36,5 & 47,1 & $<0,001$ * \\
\hline \multicolumn{5}{|l|}{ Informações sobre como evitar problemas bucais } \\
\hline $\operatorname{Sim}$ & 60,1 & 54,7 & 43,9 & \\
\hline Não & 39,8 & 45,3 & 56,1 & $<0,001$ * \\
\hline
\end{tabular}

(continua) 


\begin{tabular}{|c|c|c|c|c|}
\hline Variáveis independentes & $\begin{array}{c}\text { Boa } \\
(n=7.322)\end{array}$ & $\begin{array}{c}\text { Regular } \\
(n=3.284)\end{array}$ & $\begin{array}{c}\text { Ruim } \\
(\mathrm{n}=2.576)\end{array}$ & Valor de $p$ \\
\hline & $\%$ & $\%$ & $\%$ & \\
\hline \multicolumn{5}{|l|}{ Condições normativas } \\
\hline \multicolumn{5}{|c|}{ Necessidade de tratamento odontológico } \\
\hline Não & 14,4 & 10,5 & 7,4 & \\
\hline Sim & 85,6 & 89,5 & 92,6 & $<0,001$ * \\
\hline \multicolumn{5}{|c|}{ Alterações de tecido mole } \\
\hline Não & 89,4 & 88,5 & 84,1 & \\
\hline Sim & 10,6 & 11,5 & 15,9 & $<0,001$ * \\
\hline \multicolumn{5}{|c|}{ Número de dentes cariados } \\
\hline 0 & 43,1 & 36,8 & 28,1 & \\
\hline $1-3$ & 33,8 & 34,6 & 31,6 & \\
\hline 4 ou mais & 23,0 & 28,6 & 40,2 & $<0,001$ * \\
\hline \multicolumn{5}{|l|}{ Doença periodontal } \\
\hline Não & 92,8 & 92,6 & 87,1 & \\
\hline Sim & 7,2 & 7,4 & 12,9 & $<0,001$ * \\
\hline \multicolumn{5}{|c|}{ Número de dentes presentes } \\
\hline $23-32$ & 47,9 & 37,4 & 29,1 & \\
\hline $13-22$ & 23,0 & 30,4 & 33,0 & \\
\hline $3-12$ & 19,7 & 21,9 & 27,6 & \\
\hline $0-2$ & 9,4 & 10,2 & 10,3 & $<0,001$ * \\
\hline \multicolumn{5}{|c|}{ Necessidade de prótese removível } \\
\hline Não necessita & 78,5 & 70,2 & 56,6 & \\
\hline Necessita parcial & 18,9 & 25,1 & 34,0 & \\
\hline Necessita total & 2,6 & 4,7 & 9,4 & $<0,001$ * \\
\hline \multicolumn{5}{|l|}{ Uso de prótese removível } \\
\hline Não usa & 66,1 & 63,4 & 68,6 & \\
\hline Usa parcial & 21,5 & 24,6 & 21,9 & \\
\hline Usa total & 12,4 & 12,0 & 9,5 & $<0,001$ * \\
\hline \multicolumn{5}{|l|}{ Condições subjetivas } \\
\hline \multicolumn{5}{|c|}{ Autopercepção da saúde bucal } \\
\hline Otima/Boa & 55,7 & 23,0 & 13,5 & \\
\hline Regular & 33,2 & 55,8 & 29,7 & \\
\hline Péssima/Ruim & 11,1 & 21,2 & 56,8 & $<0,001$ * \\
\hline \multicolumn{5}{|l|}{ Autopercepção da dor } \\
\hline Não & 75,4 & 58,8 & 43,7 & \\
\hline Sim & 24,6 & 41,2 & 56,3 & $<0,001$ * \\
\hline \multicolumn{5}{|c|}{ Autopercepção da necessidade de tratamento } \\
\hline Não & 26,4 & 14,6 & 11,6 & \\
\hline Sim & 73,6 & 85,4 & 88,4 & $<0,001$ * \\
\hline
\end{tabular}

* Valor de p obtido por $\chi^{2}$.

gengivas nos últimos seis meses; perceber a necessidade de tratamento odontológico. Além disso, a autopercepção ruim da mastigação apresentou associação inversa com o sexo masculino e com o uso de prótese total. Já a autopercepção regular da mastigação esteve associada a todos os fatores supracitados com exceção de residir na Região Centro-oeste, ter idade entre 40-44 anos, ter menos de sete anos de escola- ridade, ter usado serviço odontológico há mais de três anos, ter alteração de tecido mole, ser portador de doença periodontal e usar prótese total (Tabela 2).

$\mathrm{Na}$ análise multivariada com o odds ratio (OR) ajustado para os fatores que compõem cada bloco de variáveis reunido por afinidade, conforme o modelo utilizado (Tabela 3), todos os fatores associados estatisticamente à autoper- 


\section{Tabela 2}

Variáveis do ambiente externo, características individuais, comportamentos em saúde bucal e condições normativas e subjetivas de saúde bucal associadas com a autopercepção regular e ruim da mastigação na análise bivariada. Projeto SBBrasil 2002-2003

\begin{tabular}{|c|c|c|}
\hline \multirow[t]{2}{*}{ Variáveis independentes } & Regular & Ruim \\
\hline & OR * (IC95\%) & OR * (IC95\%) \\
\hline \multicolumn{3}{|l|}{ Ambiente externo } \\
\hline \multicolumn{3}{|l|}{ Macrorregião } \\
\hline Sul & 1,0 & 1,0 \\
\hline Sudeste & $1,2(1,0-1,3)$ ** & $1,5(1,3-1,8)$ ** \\
\hline Centro-oeste & $1,1(0,9-1,2)$ & $1,6(1,4-1,9)$ ** \\
\hline Nordeste & $1,4(1,2-1,5) * \star$ & $2,3(2,1-2,7)$ ** \\
\hline Norte & $1,5(1,3-1,7)$ ** & $2,0(1,7-2,3)$ ** \\
\hline \multicolumn{3}{|l|}{ Local } \\
\hline Urbana & 1,0 & 1,0 \\
\hline Rural & $0,9(0,8-1,0)$ & $1,1(1,0-1,3)$ \\
\hline \multicolumn{3}{|l|}{ Sistema de atenção à saúde } \\
\hline Plano de saúde/Particular & 1,0 & 1,0 \\
\hline Público/Filantrópico & $1,2(1,1-1,3) * \star$ & $1,5(1,4-1,7)$ ** \\
\hline \multicolumn{3}{|l|}{ Características individuais } \\
\hline \multicolumn{3}{|l|}{ Idade (anos) } \\
\hline $35-39$ & 1,0 & 1,0 \\
\hline $40-44$ & $1,0(1,0-1,1)$ & $1,1(1,0-1,2)$ ** \\
\hline \multicolumn{3}{|l|}{ Sexo } \\
\hline Feminino & 1,0 & 1,0 \\
\hline Masculino & $0,9(0,8-1,0) * *$ & $0,7(0,7-0,8)$ ** \\
\hline \multicolumn{3}{|l|}{ Cor de pele autodeclarada } \\
\hline Branco & 1,0 & 1,0 \\
\hline Pardo & $1,3(1,2-1,4)$ ** & $1,8(1,6-2,0)$ ** \\
\hline Negro & $1,2(1,0-1,4) * *$ & $1,7(1,4-2,0)$ ** \\
\hline Amarelo e índio & $1,2(1,0-1,4)$ & $1,2(1,0-1,6)$ \\
\hline \multicolumn{3}{|l|}{ Escolaridade (anos de estudo) } \\
\hline 8 ou mais & 1,0 & 1,0 \\
\hline $4-7$ & $1,0(0,9-1,1)$ & $1,4(1,2-1,5)$ ** \\
\hline $0-3$ & $1,0(1,0-1,2)$ & $2,2(2,0-2,5)$ ** \\
\hline \multicolumn{3}{|l|}{ Renda per capita (\% do salário mínimo/dólar) } \\
\hline$\geq 68 \%(\geq$ US $\$ 50,00)$ & 1,0 & 1,0 \\
\hline$\geq 31 \%$ e $<68 \%(\geq$ US $\$ 22,33$ e $<$ US $\$ 50,00)$ & $1,3(1,2-1,5) \star \star$ & $1,8(1,6-2,0)$ ** \\
\hline$<31 \%(<$ US $\$ 22,33)$ & 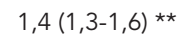 & $2,5(2,2-2,7)^{\star \star}$ \\
\hline \multicolumn{3}{|l|}{ Comportamento } \\
\hline \multicolumn{3}{|l|}{ Tempo de uso dos serviços odontológicos (anos) } \\
\hline 3 ou menos & 1,0 & 1,0 \\
\hline Mais de 3 & $1,0(1,0-1,1)$ & $1,6(1,5-1,8) * \star$ \\
\hline \multicolumn{3}{|l|}{ Informações sobre como evitar problemas bucais } \\
\hline Sim & 1,0 & 1,0 \\
\hline Não & $1,2(1,1-1,3)$ ** & $1,9(1,8-2,1)$ ** \\
\hline
\end{tabular}

(continua) 
Tabela 2 (continuação)

\begin{tabular}{|c|c|c|}
\hline \multirow[t]{2}{*}{ Variáveis independentes } & Regular & Ruim \\
\hline & OR * (IC95\%) & OR * (IC95\%) \\
\hline \multicolumn{3}{|l|}{ Condições normativas } \\
\hline \multicolumn{3}{|c|}{ Necessidade de tratamento odontológico } \\
\hline Não & 1,0 & 1,0 \\
\hline Sim & $1,4(1,2-1,6)$ ** & $2,1(1,8-2,5) * *$ \\
\hline \multicolumn{3}{|l|}{ Alterações de tecido mole } \\
\hline Não & 1,0 & 1,0 \\
\hline Sim & $1,1(1,0-1,2)$ & $1,6(1,4-1,8) \star \star$ \\
\hline \multicolumn{3}{|c|}{ Número de dentes cariados } \\
\hline 0 & 1,0 & 1,0 \\
\hline $1-3$ & $1,2(1,0-1,3) * *$ & $1,4(1,3-1,6) * \star$ \\
\hline 4 ou mais & $1,4(1,3-1,6) * \star$ & $2,7(2,4-3,0) \star \star$ \\
\hline \multicolumn{3}{|l|}{ Doença periodontal } \\
\hline Não & 1,0 & 1,0 \\
\hline Sim & $1,0(0,8-1,3)$ & $1,9(1,5-2,5) * \star$ \\
\hline \multicolumn{3}{|c|}{ Número de dentes permanentes } \\
\hline 23-32 & 1,0 & 1,0 \\
\hline $13-22$ & $1,7(1,5-1,9)$ ** & $2,4(2,1-2,6) * *$ \\
\hline $3-12$ & $1,4(1,3-1,6) * *$ & $2,3(2,0-2,6) * *$ \\
\hline $0-2$ & $1,4(1,2-1,6) * *$ & $1,8(1,5-2,1)^{\star \star}$ \\
\hline \multicolumn{3}{|c|}{ Necessidade de prótese removível } \\
\hline Não necessita & 1,0 & 1,0 \\
\hline Necessita parcial & $1,5(1,3-1,6) * *$ & 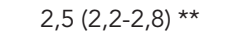 \\
\hline Necessita total & $2,0(1,6-2,5)$ ** & $5,1(4,2-6,2) * \star$ \\
\hline \multicolumn{3}{|l|}{ Uso de prótese removível } \\
\hline Não usa & 1,0 & 1,0 \\
\hline Usa parcial & $1,2(1,1-1,3)$ ** & $1,0(0,9-1,1)$ \\
\hline Usa total & $1,0(0,9-1,2)$ & $0,7(0,6-0,9) * *$ \\
\hline \multicolumn{3}{|l|}{ Condições subjetivas } \\
\hline \multicolumn{3}{|c|}{ Autopercepção da saúde bucal } \\
\hline Ótima/Boa & 1,0 & 1,0 \\
\hline Regular & $4,1(3,7-4,5)$ ** & 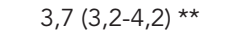 \\
\hline Ruim/Péssima & $4,6(4,1-5,2) * \star$ & $21,1(18,3-24,3)^{* *}$ \\
\hline \multicolumn{3}{|l|}{ Autopercepção da dor } \\
\hline Não & 1,0 & 1,0 \\
\hline Sim & $2,1(2,0-2,3)$ ** & $3,9(3,6-4,3) * \star$ \\
\hline \multicolumn{3}{|c|}{ Autopercepção da necessidade de tratamento } \\
\hline Não & 1,0 & 1,0 \\
\hline Sim & $2,1(1,9-2,3) * \star$ & $2,7(2,4-3,1) * \star$ \\
\hline
\end{tabular}

IC95\%: intervalo de 95\% de confiança; OR: odds ratio.

* OR obtido por regressão logística multinomial, tendo, como categoria de referência, a percepção da mastigação como boa; ${ }^{* *} p<0,05$.

cepção ruim da mastigação na análise bivariada permaneceram na análise por blocos, sendo que as magnitudes das associações observadas diminuíram em sua maioria. No bloco de condições normativas, observa-se, após o ajuste, um gradiente dose-resposta inverso na associação entre número de dentes presentes na boca e au- topercepção ruim da mastigação. Com relação às variáveis associadas à autopercepção regular da mastigação, as seguintes variáveis perdem significância após o ajuste por bloco: escolaridade, tempo de uso de serviços odontológicos, alteração de tecido mole e uso de prótese removível (Tabela 3). 


\section{Tabela 3}

Resultados da análise multivariada dos fatores associados à autopercepção da mastigação em adultos em cada um dos blocos de variáveis reunidos por afinidade. Projeto SBBrasil 2002-2003.

\begin{tabular}{|c|c|c|}
\hline Variáveis independentes & $\begin{array}{c}\text { Regular } \\
\text { OR ajustado * (IC95\%) }\end{array}$ & $\begin{array}{c}\text { Ruim } \\
\text { OR ajustado * (IC95\%) }\end{array}$ \\
\hline \multicolumn{3}{|l|}{ Ambiente externo } \\
\hline \multicolumn{3}{|l|}{ Macrorregião } \\
\hline Sul & 1,0 & 1,0 \\
\hline Sudeste & $1,1(1,0-1,3)$ & 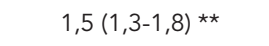 \\
\hline Centro-oeste & $1,1(1,0-1,3)$ & $1,7(1,4-2,0) \star \star$ \\
\hline Nordeste & $1,3(1,2-1,5)$ ** & $2,3(2,0-2,6) * \star$ \\
\hline Norte & $1,5(1,3-1,7) * \star$ & $1,9(1,6-2,2) * *$ \\
\hline \multicolumn{3}{|l|}{ Sistema de atenção à saúde } \\
\hline Plano de saúde/Particular & 1,0 & 1,0 \\
\hline Público/Filantrópico & $1,2(1,1-1,3)$ ** & $1,5(1,3-1,6) * *$ \\
\hline \multicolumn{3}{|l|}{ Características individuais } \\
\hline \multicolumn{3}{|l|}{ Idade (anos) } \\
\hline $35-39$ & 1,0 & 1,0 \\
\hline $40-44$ & $1,1(1,0-1,2)$ & $1,1(1,0-1,2) * \star$ \\
\hline \multicolumn{3}{|l|}{ Sexo } \\
\hline Feminino & 1,0 & 1,0 \\
\hline Masculino & $0,9(0,8-1,0) \star \star$ & $0,7(0,6-0,8) * \star$ \\
\hline \multicolumn{3}{|l|}{ Cor de pele autodeclarada } \\
\hline Branco & 1,0 & 1,0 \\
\hline Pardo & $1,2(1,1-1,3) * \star$ & $1,5(1,4-1,7) * \star$ \\
\hline Negro & $1,1(1,0-1,3)$ & $1,3(1,1-1,6)$ ** \\
\hline Amarelo e índio & $1,2(0,9-1,4)$ & $1,1(0,9-1,5)$ \\
\hline \multicolumn{3}{|l|}{ Escolaridade (anos de estudo) } \\
\hline 8 ou mais & 1,0 & 1,0 \\
\hline $4-7$ & $0,9(0,8-1,0)$ & $1,2(1,0-1,3) * *$ \\
\hline $0-3$ & $0,9(0,8-1,0)$ & $1,6(1,4-1,8) * *$ \\
\hline \multicolumn{3}{|l|}{ Renda per capita (\% do salário mínimo/dólar) } \\
\hline$\geq 68 \%(\geq$ US $\$ 50,00)$ & 1,0 & 1,0 \\
\hline$\geq 31 \%$ e $<68 \%(\geq$ US $\$ 22,33$ e $<$ US $\$ 50,00)$ & 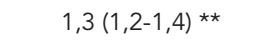 & $1,5(1,3-1,7) * \star$ \\
\hline$<31 \%(<$ US $\$ 22,33)$ & 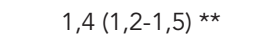 & $1,8(1,6-2,0) * \star$ \\
\hline \multicolumn{3}{|l|}{ Comportamento } \\
\hline \multicolumn{3}{|l|}{ Tempo de uso dos serviços odontológicos (anos) } \\
\hline 3 ou menos & 1,0 & 1,0 \\
\hline Mais de 3 anos & $1,0(0,9-1,1)$ & $1,5(1,3-1,6)$ \\
\hline \multicolumn{3}{|l|}{ Informações sobre como evitar problemas bucais } \\
\hline $\operatorname{Sim}$ & 1,0 & 1,0 \\
\hline Não & $1,2(1,1-1,4) * *$ & $1,8(1,6-2,0) * \star$ \\
\hline
\end{tabular}

(continua) 
Tabela 3 (continuação)

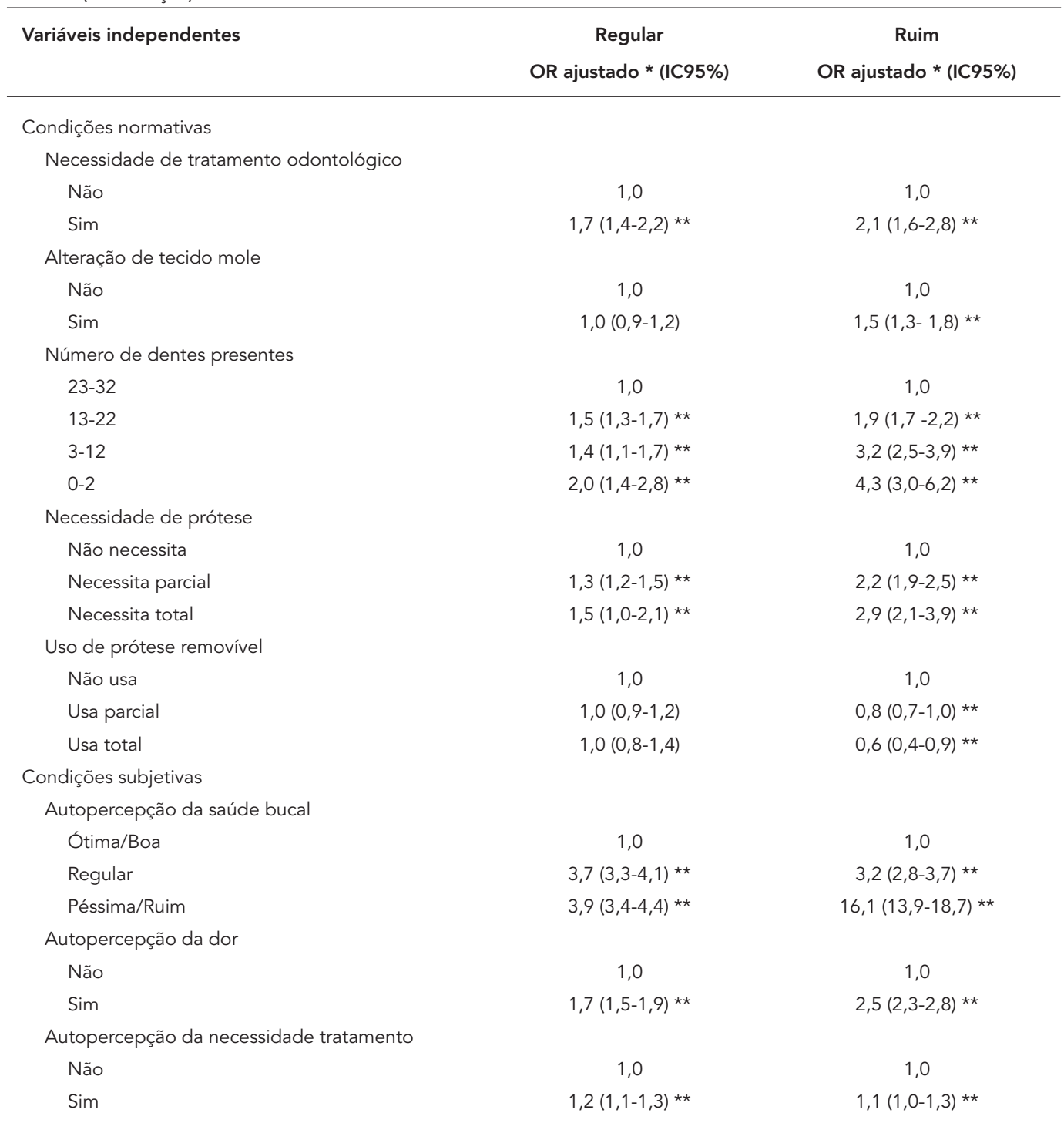

IC95\%: intervalo de 95\% de confiança; OR: odds ratio.

* OR ajustado pelas variáveis pertencentes a um mesmo bloco: ambiente externo, características individuais, comportamento, condições normativas e condições subjetivas; OR obtido por regressão logística multinomial, tendo, como categoria de referência, a percepção da mastigação como boa;

** $p<0,05$.

Todas as variáveis que foram associadas significativamente com a autopercepção regular ou ruim da mastigação na análise intermediária por bloco de afinidades foram incluídas no modelo final. Os resultados mostram que há algumas diferenças entre as variáveis associadas à autopercepção regular e ruim da mastigação. Os fatores associados tanto à autopercepção ruim como à regular da mastigação foram: residir na Região Norte; não ter recebido informações sobre como evitar problemas bucais; ter menos de 23 dentes remanescentes; necessitar de prótese parcial ou total; perceber a saúde bucal como regular, ruim ou péssima; relatar dor em dentes e gengivas e relatar a necessidade de tratamento odontológico. Residir fora da Região Sul, ser mulher e se autodeclarar pardo são fatores que estiveram associados somente à autopercepção ruim da mastigação, enquanto usar prótese parcial ou total diminuiu a chance dessa classificação. Ter 7 anos ou menos de estudo apresentou associação com a autopercepção regular da mastigação, enquanto ter consultado o dentista há mais de 3 anos reduziu a chance em classificar a mastigação como regular (Tabela 4). 


\section{Discussão}

Cerca de um quinto dos adultos percebeu a mastigação como ruim, e um quarto, como regular. Essas prevalências são elevadas em relação às taxas de prevalência de insatisfação ou dificuldades mastigatórias em estudos internacionais 7,8,9, e, em conjunto, a prevalência de autopercepção da mastigação regular e ruim foi próxima à taxa encontrada na população idosa brasileira ${ }^{6}$. Tais resultados são preocupantes, pois a insatisfação mastigatória está vinculada a restrições alimentares e piora na qualidade de vida $6,7,8,9,17$.

Como visto em outros estudos, uma multiplicidade de fatores contribui para a insatisfação com a mastigação $6,7,8,9,18,19$, o que corrobora a utilização de um modelo multidimensional para a análise dos fatores associados à percepção da mastigação regular e ruim no presente trabalho. Alguns estudos sobre o tema utilizaram modelos hierárquicos e multidimensionais $6,7,8,9$, porém o modelo proposto por Gift et al. 16, adaptado por Martins et al. 12, foi o que contemplou domínios mais abrangentes, sendo, por isso, $\mathrm{o}$ adotado.

Considerando a Região Sul do país como referência, a chance de perceber a mastigação como ruim foi maior em todas as demais regiões, mostrando a abrangência desse problema para a saúde pública em nível nacional e indicando, ao mesmo tempo, a presença de desigualdade regional que necessita ser superada.

Ser mulher e se autodeclarar pardo foram as características individuais associadas à autopercepção ruim da mastigação. A associação com o sexo não foi encontrada em outros estudos internacionais 7,8,9. Uma hipótese para esse resultado seria a de que, no Brasil, as mulheres apresentam maior prevalência de perdas dentárias do que os homens 20 . Além disso, um estudo realizado por Locker \& Miller 9 , no Canadá, encontrou que elas também apresentam maior preocupação com aparência e saúde dos dentes e da boca. Apesar de a análise ter sido ajustada por alguns desses fatores, é possível que diferenças residuais entre homens e mulheres com relação a essas variáveis tenham permanecido e expliquem as diferenças observadas na análise final.

Com relação à raça ou cor de pele autodeclarada, é difícil fazer uma comparação exata, pois os estudos ou não analisam essa característica 9,18 ou utilizaram a dicotomização de cor/ raça em brancos e negros, sendo a prevalência de limitações mastigatórias maior em indivíduos negros 7,8. Somente o estudo realizado com idosos brasileiros utilizou a mesma classificação e verificou maior prevalência de insatisfação mastigatória em quem se autodeclarou pardo ou negro ${ }^{6}$. Brasileiros pardos e negros apresentam maior prevalência de perdas dentárias do que os brancos 20 , e isso pode ser uma explicação para os resultados encontrados, muito embora não tenha sido encontrada associação entre cor da pele negra e autopercepção ruim da mastigação.

Ter escolaridade igual a ou menor que 7 anos foi o fator sociodemográfico que esteve associado somente à autopercepção regular da mastigação. Entretanto, diferentemente do esperado, a menor escolaridade associou-se negativamente à autopercepção regular da mastigação, o que discorda de estudos internacionais que verificaram que, quanto mais baixo o nível educacional, maior a insatisfação com a mastigação $7,8,9,10,11,12,13,14,15,17$. Uma explicação para esse fato pode ser a resignação de pessoas menos escolarizadas no Brasil, uma vez que apenas recentemente, com a instituição do programa Brasil Sorridente no âmbito do SUS (Ministério da Saúde. Brasil Sorridente http://portal.saude.gov.br/ portal/saude/area.cfm?id_area $=406$, acessado em 20/Fev/2011), a saúde bucal da população brasileira passou a ser priorizada. No entanto, o programa está apenas iniciando, e as consequências da carência histórica de assistência bucal são inestimáveis e muitas delas, irreversíveis. Como o valor de $\mathrm{p}$ da associação da menor escolaridade com a mastigação regular foi borderli$n e$, é possível que ainda haja confusão residual devido à presença de outros indicadores sociais e econômicos não utilizados ou aos pontos de corte empregados na análise.

O tempo de uso do serviço odontológico esteve associado somente à autopercepção regular da mastigação e de forma inversa, ou seja, os indivíduos que consultaram o dentista há mais de 3 anos tiveram menor chance em perceber a mastigação como regular. Gilbert et al. 7 verificaram que a prevalência de limitações na mastigação foi muito maior em indivíduos que procuraram o dentista somente ao apresentarem algum problema, comparados àqueles que vão regularmente ao dentista. $O$ resultado da análise apresentada neste artigo é difícil de explicar, visto que o tempo de uso de serviços de saúde não foi associado à autopercepção ruim da mastigação. Não é possível excluir a possibilidade de que a busca pelo serviço expresse uma situação emergencial apenas, associada à dor e à perda dentária, visto que geralmente o serviço dentário era pago ou muito precário e limitado apenas a indivíduos muito carentes. Nesse sentido, a frequência ao serviço odontológico mais recente incluiria um percentual grande de indivíduos que buscaram o serviço quando estavam em grande necessidade, muitas vezes em condições que já comprometiam sua saúde bucal, inclusive a mastigação. 
Fatores associados à autopercepção da mastigação em adultos no modelo final. Projeto SBBrasil 2002-2003.

\begin{tabular}{|c|c|c|}
\hline Variáveis independentes & $\begin{array}{c}\text { Regular } \\
\text { OR ajustado * (IC95\%) }\end{array}$ & $\begin{array}{c}\text { Ruim } \\
\text { OR ajustado * (IC95\%) }\end{array}$ \\
\hline \multicolumn{3}{|l|}{ Ambiente externo } \\
\hline \multicolumn{3}{|l|}{ Macrorregião } \\
\hline Sul & 1,0 & 1,0 \\
\hline Sudeste & $1,2(1,0-1,4)$ & $1,2(1,0-1,5) * \star$ \\
\hline Centro-oeste & $1,0(0,9-1,2)$ & $1,3(1,0-1,6) * \star$ \\
\hline Nordeste & $1,2(1,0-1,4)$ & $1,5(1,2-1,8)$ ** \\
\hline Norte & $1,3(1,1-1,5)$ ** & $1,3(1,0-1,6)$ ** \\
\hline \multicolumn{3}{|l|}{ Características individuais } \\
\hline \multicolumn{3}{|l|}{ Sexo } \\
\hline Feminino & 1,0 & 1,0 \\
\hline Masculino & $0,9(0,8-1,0)$ & $0,8(0,7-0,9) * \star$ \\
\hline \multicolumn{3}{|c|}{ Cor de pele autodeclarada } \\
\hline Branco & 1,0 & 1,0 \\
\hline Pardo & $1,1(1,0-1,2)$ & $1,3(1,1-1,5) * *$ \\
\hline Negro & $1,1(0,9-1,3)$ & $1,2(1,0-1,5)$ \\
\hline Amarelo e índio & $1,1(0,8-1,4)$ & $1,2(0,9-1,7)$ \\
\hline \multicolumn{3}{|c|}{ Escolaridade (anos de estudo) } \\
\hline 8 ou mais & 1,0 & 1,0 \\
\hline $4-7$ & $0,9(0,8-1,0) * *$ & $0,9(0,8-1,0)$ \\
\hline $0-3$ & $0,8(0,7-1,0) * *$ & $1,1(1,0-1,3)$ \\
\hline \multicolumn{3}{|l|}{ Comportamento } \\
\hline \multicolumn{3}{|c|}{ Tempo de uso dos serviços odontológicos (anos) } \\
\hline 3 ou menos & 1,0 & 1,0 \\
\hline Mais de 3 & $0,8(0,7-0,9) * \star$ & $1,0(0,9-1,1)$ \\
\hline \multicolumn{3}{|c|}{ Informações sobre como evitar problemas bucais } \\
\hline Sim & 1,0 & 1,0 \\
\hline Não & $1,1(1,0-1,2)$ ** & $1,3(1,2-1,5) * \star$ \\
\hline \multicolumn{3}{|l|}{ Condições normativas } \\
\hline \multicolumn{3}{|c|}{ Número de dentes presentes } \\
\hline $23-32$ & 1,0 & 1,0 \\
\hline $13-22$ & $1,5(1,3-1,7)$ ** & $1,9(1,6-2,2) * \star$ \\
\hline $3-12$ & $1,5(1,1-1,8)$ ** & $3,2(2,5-4,1) * \star$ \\
\hline $0-2$ & $2,3(1,6-3,3) * \star$ & $5,6(3,8--8,4)$ ** \\
\hline \multicolumn{3}{|c|}{ Necessidade de prótese removível } \\
\hline Não necessita & 1,0 & 1,0 \\
\hline Necessita parcial & $1,2(1,1-1,4) * *$ & $1,5(1,3-1,8) * *$ \\
\hline Necessita total & $1,6(1,1-2,4) * *$ & $2,7(1,8-3,7)$ ** \\
\hline \multicolumn{3}{|l|}{ Uso de prótese removível } \\
\hline Não usa & 1,0 & 1,0 \\
\hline Usa parcial & $1,0(0,8-1,1)$ & $0,8(0,7-1,0)$ ** \\
\hline Usa total & $1,0(0,7-1,4)$ & $0,5(0,3-0,7)$ ** \\
\hline
\end{tabular}

(continua) 


\begin{tabular}{|c|c|c|}
\hline \multirow[t]{2}{*}{ Variáveis independentes } & Regular & Ruim \\
\hline & OR ajustado * (IC95\%) & OR ajustado * (IC95\%) \\
\hline \multicolumn{3}{|l|}{ Condições subjetivas } \\
\hline \multicolumn{3}{|c|}{ Autopercepção da saúde bucal } \\
\hline Ótima/Boa & 1,0 & 1,0 \\
\hline Regular & $3,6(3,2-4,0) * \star$ & $3,4(2,9-4,0) * \star$ \\
\hline Péssima/Ruim & $3,7(3,1-4,3) * \star$ & $15(12,4-17,7)$ ** \\
\hline \multicolumn{3}{|l|}{ Autopercepção da dor } \\
\hline Não & 1,0 & 1,0 \\
\hline Sim & $1,8(1,6-2,0) * \star$ & $2,8(2,4-3,1) * \star$ \\
\hline \multicolumn{3}{|c|}{ Autopercepção da necessidade tratamento } \\
\hline Não & 1,0 & 1,0 \\
\hline Sim & $1,3(1,1-1,5)$ ** & $1,2(1,0-1,5)$ ** \\
\hline
\end{tabular}

IC95\%: intervalo de 95\% de confiança; OR: odds ratio.

* OR ajustado por todas as variáveis com $p<0,05$ no teste de regressão logística multinomial, tendo, como categoria de referência, a percepção da mastigação como boa;

** $p<0,05$.

A falta de acesso às informações sobre como evitar os problemas bucais esteve associada à autopercepção ruim da mastigação neste estudo, mostrando a lacuna presente na atenção básica de saúde bucal quanto às ações educativas. Ter informações sobre problemas bucais é importante, pois a perda dentária associada ao avanço da idade diminui com a melhoria das condições de vida, de higiene e de cuidados gerais e bucais 21,22 . Uma pesquisa verificou que indivíduos que utilizam o sistema público têm maiores chances de não receber informações sobre como evitar problemas bucais, comparados àqueles que utilizam o sistema privado 23 . Nesse estudo, entretanto, o tipo de sistema de saúde utilizado não foi associado à percepção ruim da mastigação no modelo final.

As perdas dentárias estão diretamente relacionadas à piora da mastigação 6,7,8,9,18,24,25. No presente estudo, o número de dentes presentes e a necessidade de prótese estiveram fortemente associados à autopercepção ruim da mastigação, com claros gradientes dose-resposta em relação tanto à diminuição do número de dentes quanto à necessidade de prótese parcial e total. Os contatos dentários são essenciais para a realização da incisão, trituração e pulverização do alimento, e a diminuição desses interfere no padrão e força mastigatória, sendo a função mastigatória também importante para manutenção da estabilidade dentária 1. A alta prevalência de perdas dentárias verificadas no presente estudo e sua forte relação com a insatisfação mastigatória devem servir como alerta aos serviços de saúde pública sobre a necessidade de condutas preventivas de cuidados dentários 25,26, além de mostrar a necessidade de ampliar a oferta e melhorar o acesso a procedimentos mais especializados, como a prótese dentária parcial e total, para aqueles que necessitam.

No presente estudo, o uso da prótese, tanto parcial como total, reduziu a chance de os indivíduos perceberem a mastigação como ruim, em especial, o uso de prótese total. Apesar de apresentar menor eficiência mastigatória e força de mordida, a prótese total foi avaliada positivamente por seus usuários ao restabelecer a função mastigatória 27 , o que também foi encontrado em outros estudos 18,28,29. Classificar a mastigação como "boa” também pode estar relacionado ao tempo de uso da prótese 18,29 , ao tipo 30 e à qualidade da prótese 29. Estudo verificou que, quanto maior o tempo com a prótese, maior a satisfação com a mastigação 18 . Atualmente, verifica-se que a prótese sobre implantes dentários é a que está associada à maior satisfação mastigatória ${ }^{30}$, porém esse procedimento ainda não é disponibilizado pelo sistema público de saúde, possivelmente por ser tratar de um tratamento de alto custo 31 .

Em relação às condições subjetivas, a autopercepção ruim ou péssima da saúde bucal, a dor e a autopercepção da necessidade de tratamento apresentaram-se fortemente associadas à autopercepção ruim da mastigação, sendo mais expressiva a associação com a autopercepção da saúde bucal. A associação direta entre os diversos itens de autopercepção da saúde bucal também 
foi verificada em outros estudos 7,8,9,12,18. É esperado que a saúde bucal e a mastigação estejam fortemente associadas, uma vez que estão relacionadas às mesmas estruturas. Alterações no sistema mastigatório refletem problemas sociais e de saúde, seja bucal ou geral, como restringir a alimentação junto a outras pessoas 8 e evitar determinados tipos de alimentos, principalmente vegetais, levando ao aumento do risco de doença cardiovascular, entre outras 14 .

A presença de dor durante a mastigação pode alterar o padrão mastigatório e a ingestão de determinados alimentos importantes para a saúde, como aqueles ricos em fibras, interferindo na saúde geral do indivíduo, na funcionalidade da musculatura envolvida e da articulação temporomandibular ou ser consequência de alterações nessas estruturas 2,4,5. É importante citar que a dor decorrente da disfunção da articulação temporomandibular não foi contemplada no SBBrasil, porém a resposta da presença de dor em dentes e gengivas pode se confundir com a dor decorrente da disfunção da articulação temporomandibular, uma vez que essa é muito prevalente em adultos e pode irradiar-se para dentes e gengivas 32 . A percepção da necessidade de tratamento odontológico esteve positivamente associada à autopercepção regular e ruim da mastigação no modelo final, mostrando que os indivíduos reconhecem a presença de problemas dentários, e que esses estão associados a outras queixas bucais.

O delineamento transversal do presente estudo permite conhecer como a população adulta no país percebe sua mastigação e identificar fatores associados a essa percepção. O SBBrasil constitui-se em uma importante base de dados para conhecer a saúde bucal da população brasileira pelo grande número de participantes, pela qualidade geral e diversidade das informações obtidas e pela contemplação de todas as regiões do país. Há, entretanto, um questionamento se a amostra do SBBrasil representa a população brasileira, especialmente pela ausência de correção pelo desenho amostral 33. Narvai et al. 34 constataram que o impacto da não atribuição de pesos amostrais sobre as medidas de prevalência é pequeno, e o efeito de correções de desenho amostral sobre as medidas de associação é ainda menor, correspondendo a alterações em casas decimais, sem impacto na magnitude nem na direção das associações encontradas. É importante ressaltar ainda que, por se tratar de um estudo transversal, não é possível estabelecer relações causais entre os fatores de interesse investigados e a autopercepção regular ou ruim da mastigação.

Como mostram resultados de pesquisas 11,17, a piora da mastigação está relacionada à piora na qualidade de vida, sendo assim, políticas de saúde bucal voltadas para a população adulta poderão melhorar a condição mastigatória e a qualidade de vida dos adultos, que constituem a população economicamente ativa do país. Os profissionais que atuam direta ou indiretamente com a saúde bucal, como cirurgiões-dentistas e fonoaudiólogos, deverão trabalhar cientes de que os problemas na mastigação não se restringem aos danos bucais localizados, mas afetam também o convívio social e a qualidade de vida dos indivíduos.

A partir dos resultados deste estudo, observou-se que uma parcela considerável da população adulta brasileira classifica a sua mastigação como regular ou ruim, e que fatores objetivos e subjetivos, ambientais e pessoais associam-se a essa autopercepção. 


\section{Resumo}

Propôs-se estimar a prevalência da autopercepção da mastigação e investigar os fatores relacionados à autopercepção regular e ruim. Foram entrevistados e examinados 13.431 adultos, participantes do projeto SBBrasil. Foram realizadas análises descritiva, bivariada $\left(\chi^{2} ; p<0,20\right)$ e multivariada por meio de regressão logística multinomial ( $p<0,05)$, com IC95\%. 55,6\% classificaram a mastigação como boa; $24,9 \%$, como regular; e 19,5\%, como ruim. Na analise bivariada, todos os fatores foram associados à autopercepção da mastigação regular ou ruim $(p<0,20)$. No modelo final, os fatores associados tanto com a percepção regular como ruim foram: não receber informações sobre como evitar problemas bucais, apresentar menos de 23 dentes remanescentes, necessitar de prótese parcial ou total, perceber a saúde bucal como regular ou ruimpéssima, relatar dor em dentes e gengivas e necessidade de tratamento odontológico. Conclui-se que diversos fatores associam-se à autopercepção da mastigação, principalmente as condições subjetivas, reforçando sua importância como indicador em saúde bucal.

Mastigação; Sáude Bucal; Adulto

\section{Colaboradores}

A. P. G. Braga idealizou o trabalho, realizou a revisão de literatura, auxiliou na análise dos dados, redigiu a primeira versão do manuscrito, participou da discussão e da redação final. S. M. Barreto idealizou o trabalho, trabalhou na análise dos dados, revisão do manuscrito, discussão e participou da redação final. A. M. E. B. L. Martins idealizou o trabalho, contribuiu no fornecimento e análise dos dados, revisão e redação final.

\section{Agradecimentos}

À Coordenação de Aperfeiçoamento de Pessoal de Nível Superior (CAPES) pelo financiamento.

\section{Referências}

1. Douglas CR. Fisiologia da mastigação. In: Douglas CR, organizador. Tratado de fisiologia aplicada à fonoaudiologia. São Paulo: Robe Editorial; 2002. p. 345-68.

2. Bianchini EMG. Mastigação e ATM. In: Bianchini EMG, organizador. Fundamentos em fonoaudiologia: aspectos clínicos em motricidade orofacial. 2a Ed. Rio de Janeiro: Editora Guanabara Koogan; 2005. p. 45-58.

3. Enlow DH, Hans MG. Noções básicas sobre crescimento facial. São Paulo: Livraria Santos Editora; 2002.

4. Ferreira CLP, Silva MAR, Felício CM. Orofacial myofunctional disorder in subjects with temporomandibular disorder. Cranio 2009; 27:268-74.

5. Felício CM, Melchior MO, Silva MAMR, Celeghini RMS. Desempenho mastigatório em adultos relacionado com a desordem temporomandibular e com a oclusão. Pró-Fono 2007; 19:151-8.

6. Dias-Costa JS, Galli R, Oliveira EA, Backes V, Vial EA, Canuto R, et al. Prevalência de capacidade mastigatória insatisfatória e fatores associados em idosos brasileiros. Cad Saúde Pública 2010; 26 : 79-88.
7. Gilbert GH, Foerster U, Duncan, RP. Satisfaction with chewing ability in a diverse sample of dentate adults. J Oral Rehabil 1998; 25:15-27.

8. Gilbert GH, Duncan RP, Heft MW, Dolan TA, Vogel WB. Oral disadvantage among dentate adults. Community Dent Oral Epidemiol 1997; 25:301-13.

9. Locker D, MillerY. Subjectively reported oral health status in an adult population. Community Dent Oral Epidemiol 1994; 22:425-30.

10. Narvai PC, Antunes JLF. Saúde bucal: a autopercepção da mutilação e das incapacidades. In: Lebrão ML, Duarte YAO, organizadores. SABE: Saúde, bem-estar e envelhecimento. O Projeto SABE no Município de São Paulo: uma abordagem inicial. Brasília: Organização Pan-Americana da Saúde; 2003. p. 121-40.

11. Hugo FN, Hilgert JB, Sousa ML, Cury JA. Oral status and its association with general quality of life in older independent-living south-Brazilians. Community Dent Oral Epidemiol 2009; 37:231-40.

12. Martins AMEBL, Barreto SM, Pordeus IM. Autoavaliação da saúde bucal em idosos: análise com base em modelo multidimensional. Cad Saúde Pública 2009; 25:421-35. 
13. Ansai T, Takata Y, Soh I, Akifusa S, Sogame A, Shimada N, et al. Relationship between chewing ability and 4-year mortality in a cohort of 80-year-old Japanese people. Oral Dis 2007; 13:214-9.

14. Ansai T, Takata Y, Soh I, Yoshida A, Hamasaki T, Awano S, et al. Association of chewing ability with cardiovascular disease mortality in the 80-year-old Japanese population. Eur J Cardiovasc Prev Rehabil 2008; 15:104-6.

15. Coordenação Nacional de Saúde Bucal, Departamento de Atenção Básica, Secretaria de Atenção à Saúde, Ministério da Saúde. Projeto SB Brasil 2003: condições de saúde bucal da população brasileira 2002-2003. Resultados principais. Brasília: Ministério da Saúde; 2004.

16. Gift HC, Atchison KA, Drury TF. Perceptions of the natural dentition in the context of multiple variables. J Dent Res 1998; 77:1529-38.

17. Tsakos G, Sheiham A, Ilffe S, Kharicha K, Harari D, Swift CG, et al. The impact of educational level on oral health-related quality of life in older people in London. Eur J Oral Sci 2009; 117:286-92.

18. Johansson A, Unell L, Johansson AK, Carlsson GE. A 10-year longitudinal study of self-assessed chewing ability and dental status in 50-year-old subjects. Int J Prosthodont 2007; 20:643-5.

19. Hjern A, Grindefjord M, Sundberg H, Rosén M. Social inequality in oral health and use of dental care in Sweden. Community Dent Oral Epidemiol 2001; 29:167-74.

20. Barbato PR, Nagano HCM, Zanchet FN, Boing AF, Peres MA. Perdas dentárias e fatores sociais, demográficos e de serviços associados em adultos brasileiros: uma análise dos dados do Estudo Epidemiológico Nacional (Projeto SB Brasil 20022003). Cad Saúde Pública 2007; 23:1803-14.

21. Osterberg T, Carlsson GE, Sundh W, Fyhrlund A. Prognosis of and factors associated with dental status in the adult Swedish population, 1975-1989. Community Dent Oral Epidemiol 1995; 23:232-6.

22. Gilbert GH, Miller MK, Duncan P, Ringelbrg ML, Dolan TA, Forester U. Tooth-specific and personlevel predictors of 24-month tooth loss among older adults. Community Dent Oral Epidemiol 1999; 27:372-85.

23. Camargo MBJ, Dumith SC, Barros AJD. Uso regular de serviços odontológicos entre adultos: padrões de utilização e tipos de serviços. Cad Saúde Pública 2009; 25:1894-906.
24. Ferreira AAA, Piuvezan G, Werner CWA, Alves MSCF. A dor e perda dentária: representações sociais do cuidado à saúde bucal. Ciênc Saúde Coletiva 2006; 11:211-8.

25. Unfer B, Braun K, Silva CP, Pereira Filho LD. Autopercepção da perda de dentes em idosos. Interface Comun Saúde Educ 2006; 10:217-26.

26. Abegg C. Hábitos de higiene bucal de adultos porto-alegrenses. Rev Saúde Pública 1997; 31:586-93.

27. Corpas LS. Avaliação da função mastigatória associada à força de mordida e percepção oral em indivíduos usuários de prótese total [Dissertação de Mestrado]. Bauru: Faculdade de Odontologia de Bauru, Universidade de São Paulo; 2005.

28. Hassel AJ, Rolk C, Grossmann AC, Ohlmann B, Rammelsberg P. Correlations between self-ratings of denture function and oral health-related quality of life in different age groups. Int J Prosthodont 2007; 20:242-4.

29. Čelebić A, Knezović-Zlatarić D, Carek V, Baučić I, Stipetić J. Factors related to patients satisfation with complete denture therapy. J Gerontol A Biol Sci Med Sci 2003; 58:948-53.

30. Pocztaruck RL, Frasca LCF, Rivaldo EG, Mattia PRC, Vidal RA, Fernandes E, et al. Satisfaction level and mastigatory capacity in edentulous patients with conventional dentures and implant-retained overdentures. Braz J Oral Sci 2006; 5:1232-8.

31. Leles CR, Martins RR, Silva ET, Nunes MF. Discriminant analysis of patients'reasons for choosing or refusing treatments for partial edentulism. J Oral Rehabil 2009; 36:909-15.

32. Okeson JP. Tratamento das desordens temporomandibulares e oclusão. 4a Ed. São Paulo: Editora Artes Médicas; 2000.

33. Queiroz RCS, Portela MC, Vasconcellos MTL. Pesquisa sobre as Condições de Saúde Bucal da População Brasileira (SB Brasil 2003): seus dados não produzem estimativas populacionais, mas há possibilidade de correção. Cad Saúde Pública 2009; 25:47-58.

34. Narvai PC, Antunes JLF, Moysés SJ, Frazão P, Peres MA, Peres KG, et al. Validade científica de conhecimento epidemiológico gerado com base no estudo Saúde Bucal Brasil 2003. Cad Saúde Pública 2010; 26:647-70.

Recebido em 28/Abr/2011

Versão final reapresentada em 18/Nov/2011 Aprovado em 05/Jan/2012 